\title{
Modulated stellar and solar cycles: parallels and differences
}

\author{
K. Oláh ${ }^{1}$, L. van Driel-Gesztelyi $i^{1,2,3}$ and K. G. Strassmeier ${ }^{4}$ \\ ${ }^{1}$ Konkoly Observatory, Budapest, Hungary \\ email: olah@konkoly.hu \\ ${ }^{2}$ Mullard Space Science Laboratory, University College London, UK \\ ${ }^{3}$ Observatoire de Paris, LESIA, CNRS, UPMC Univ. Paris 06, Univ. Paris-Diderot, Meudon \\ Cedex, France \\ ${ }^{4}$ Leibniz Institute for Astrophysics Potsdam (AIP), Germany
}

\begin{abstract}
We present examples of activity cycle timescales on different types of stars from lowmass dwarfs to more massive giants, with wide-ranging rotation rates, and compare the observed cyclicities to the irradiance based solar cycle and its modulations. Using annual spectral solar irradiance in wavelength bands typical for stellar observations reconstructed by Shapiro et al. (2011), a direct comparison can be made between cycle timescales and amplitudes derived for the Sun and the stars. We show that cycles on multiple timescales, known to be present in solar activity, also show up on stars when the dataset is long enough to allow recognition. The cycle lengths are not fixed, but evolve - gradually during some periods but there are also changes on short timescales. In case the activity is dominated by spots, i.e., by cooler surface features, the star is redder when fainter, whereas other type of activity make the stars bluer when the activity is higher. We found the Sun to be a member of the former group, based on reconstructed spectral irradiance data by Shapiro et al. (2011).
\end{abstract}

Keywords. stars: activity, stars: spots, Sun: activity, sunspots

\section{Introduction}

The long-term variability of the Sun and other active stars' cycles is a well known feature originating from the quasi-periodic generation, evolution and decay of the magnetic field by a dynamo mechanism, which manifests itself in surface phenomena like spots and faculae. During a magnetic cycle the coverage by cooler and hotter areas of the stellar surface is changing. These changes are measured as brightness variability in case of stars, whereas for the Sun they can be traced through direct imaging. The time behavior of stellar surface phenomena is studied by searching for periodicities in the long-term brightness variation. On the Sun, the sunspot number and/or some proxy data (e.g. the $10.7 \mathrm{~cm}$ radio emission) characterize the solar cycle. For solar and stellar datasets, using time series analysis, cycles can be found. However, the amplitude of the variations depends on the observing wavelength of the datasets. In this paper we briefly describe the types of existing long-term datasets for active stars and the Sun and present a convenient and useful method to study the time-behavior of the cycles found. With the help of a recent annual spectral reconstruction of the solar irradiance variability back to centuries by Shapiro et al. (2011), we are able to make direct comparison between the long-term behavior of the solar and the stellar magnetic variability. Dynamo models indicate that the length of a cycle is determined by many factors (depth of the convection zone, rotational rate, strength of the magnetic field etc., see e.g., Brown et al. (2011)). In this paper we study the cycle features without addressing their origins. 


\section{Datasets and tools}

As the 11-yr sunspot cycle is well documented for more than 2.5 centuries, the existence of stellar cycles were postulated not long after the first observations of the active stars begun, about half a century ago. Active stars exhibit brightness variation as the spotted, cooler regions of the stellar surface are moving in and out of view due to rotation, and long-term brightness changes superposed on the rotational modulation are also present in all cases. Systematic observations of stars proposed by Wilson (1968) started in 1966 with monitoring the relative fluxes of the cores of CaII H \& K lines, which on the Sun vary with the area and intensity of chromospheric network and faculae, and thus can be used as indicators of magnetic activity in stellar chromospheres. This dataset allowed the determination of cycles on several active stars and even double cycles were found (see Baliunas et al. (1996)). Conventional photometric observations were carried out at several observatories but systematic observations of active stars only started with the advent of dedicated automated telescopes, some of those are working to date, such as e.g. the Vienna-Potsdam APT (Strassmeier et al. 1997).

Time series photometry spanning decades were gathered for a sample of active stars. We refer to Oláh et al. (2009) for details of the stellar data, but note that the data used here have been updated with data collected since then with the Vienna-Potsdam APT (Strassmeier et al. 1997). The data used are measured in $V$ color, and differential magnitudes to the comparison stars are given in the plots.

A reconstruction of the annual mean spectral irradiance of the Sun from 1610 to the present, from $130 \mathrm{~nm}$ to $10 \mu \mathrm{m}$ for every year by Shapiro et al. (2011), allows us to study the Sun as if it was measured with broadband $B V R$ filters generally used for stellar observations. The electronic tables of Shapiro et al. (2011) contain flux values of the Sun for the wavelength ranges of the above mentioned filters. Though a flux value like this is not identical to a flux which is gathered through a broadband filter with its special flux transmission, but is good enough approximation. The fluxes were transformed to magnitudes in the usual way $(-2.5 \times \log ($ flux $))$, which in this case has a zero-point error, but that does not affect the variability we are searching for in the data.

To study the cycle lengths and their evolution the method Short Term Fourier Transform (hereafter STFT) is used here. In short, the datasets are multiplied by a Gaussian window around a certain epoch, then this part is Fourier-transformed, so the frequency spectrum around that epoch is generated. Then the epoch is shifted, so a series of Fourier spectra are obtained, and a two-dimensional power spectrum can be plotted. This process needs equidistant data in time, which is valid for the solar measurements and reconstructions. For the stars the situation is different due to gaps caused by weather conditions on daily basis, and longer interruptions because of the visibility of the stars from different geographical locations. The stellar data thus need pre-processing: the rotational signal is removed from the data and after that a moving averaging and spline smoothing is applied to obtain a continuous dataset. The details and tests of the method and the pre-processing of the stellar data can be found in the paper of Kolláth \& Oláh (2009).

\subsection{The problem of the lengths of the datasets}

The longest datasets describing magnetic cycles of the closest active star, the Sun, include sunspot numbers, sunspot area, flux data determined from imaging observations, irradiance measurements, and various proxies describing the long-term changes. All these data are widely analyzed and a great number of attempts have been made to predict the activity of our star, due to its direct effect on life of Earth, see Kolláth et al. (2011). It has also been known since long, that the length of the Schwabe cycle is not constant, but 


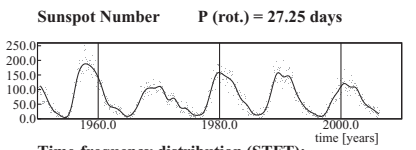

Time-frequency distribution (STFT): $\quad$ cycle period
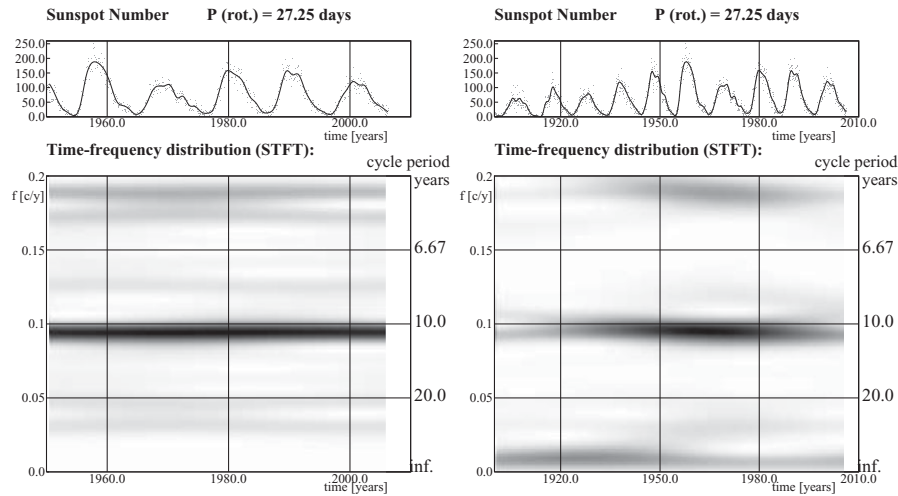

Time-frequency distribution (STFT):
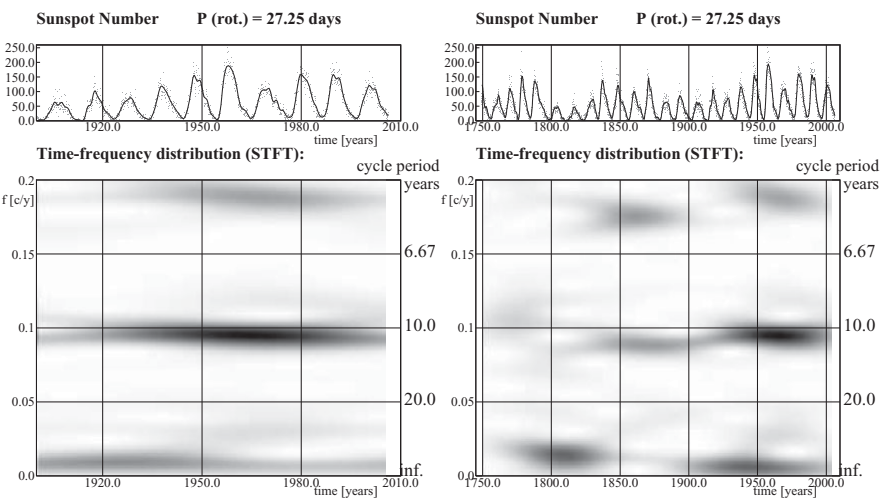

Figure 1. Time-frequency diagrams of solar activity using data for the last 50 years (left), 100 years (middle) and 250 years (right). The importance of the length of the dataset is demonstrated; from 50 years of data (which is longer than most of the stellar datasets) only the existence of the Schwabe cycle is certain. The full dataset shows an evolution of the length of the Schwabe cycle and reveals the century-long Gleissberg cycle and its time-variability.

varies between 8 and 15 years. The importance of the length of the datasets is demonstrated in Fig. 1. Time-frequency diagrams are plotted using the sunspot numbers for the last 50, 100 and 250 years, respectively. From the shortest dataset only the existence of an about 11-year long cycle is found. From the 100-year dataset an evolution in the Schwabe cycle appears, together with the sign of a changing long-term cycle. When more than 250 years of sunspot data are analyzed, a complicated variability of the Schwabe cycle and more details of the century-long Gleissberg cycle are recovered.

\section{Stellar cycles}

A comprehensive study of stellar cycles recovered from V-band photometry was published by Oláh et al. (2009) for 14 active stars, including a re-analysis of CaII data for further 6 stars. To illustrate the variety of stellar cycles, here we present time-frequency analysis of photometry for 3 stars of very different nature from the sample of Oláh et al. (2009), with the datasets now extended to 2011 (Fig. 2). The left panels are devoted to
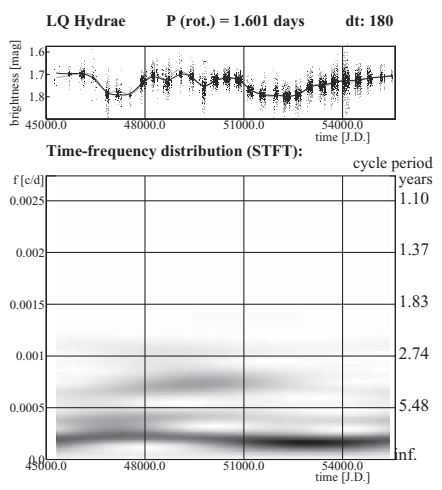
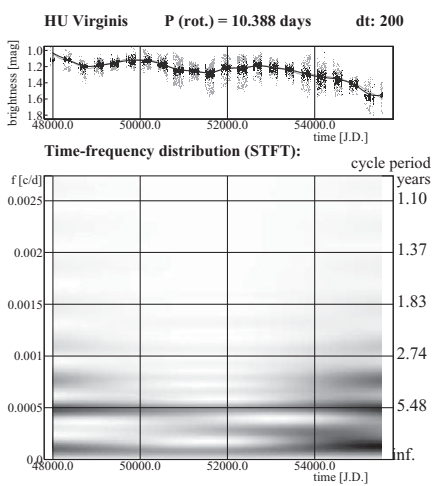
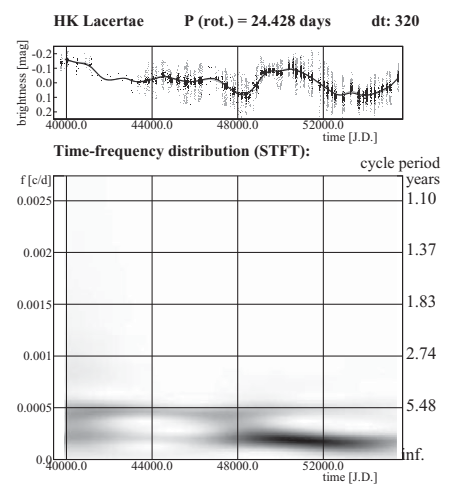

Figure 2. Long-term datasets (upper) and their time-frequency diagrams (lower) for LQ Hya (left), HU Vir (middle) and HK Lac (right). Multiple cycles showing changes both in time and amplitude are seen in all cases. In the upper panels the points represent original (red-grey) and processed (blue-black) observations, while the continuous line results from spline interpolations. For a colour figure, see the online version of the paper. 
LQ Hya; this is a fast rotating, single star of K2V type, with a rotational period of 1.601 days and with an effective temperature of about $5100 \mathrm{~K}$. The time-frequency diagram of the observations, spanning now for 28 years, clearly shows at least two, changing cycles, one on the timescale of 2-3 years and another, smoothly changing between 7 and 12 years. The middle panels of Fig. 2 display the results for HU Vir, the primary component of a synchronized binary star with an orbital and rotational period of 10.388 days. Its spectral type is K1IV-III, i.e., a subgiant with an effective temperature of $5000 \mathrm{~K}$. The 22 years long dataset, now five years longer than in Oláh et al. (2009), confirms the previously known cycle of around 5.7 years and suggests a higher amplitude of the longer term variability. The rightmost panels of Fig. 2 show the results for the K0III giant HK Lac, which is the primary of a single-lined, synchronized binary with an orbital period of 24.428 days, and an effective temperature of $4750 \mathrm{~K}$. We have the longest dataset available for this star, at present 53 years, which consists of both photometry and early photographic measurements. Cycles showing continuous changes are found around 5-6, and 10-13 years. A general decreasing trend is also evident from the data.

\section{Solar cycles}

The cycles of the Sun as conventionally treated are not fully comparable to stellar results, since the amplitudes of variability are wavelength-dependent, i.e. they are markedly different when measured from X-rays through visual-infrared to radio wavelengths. For the Sun, long series of data exist at various wavelengths, whereas long-term stellar measurements are made in a narrow vawelength range, in $B V R I$ bandpasses, i.e., between about 400-900 nm. The reconstructed spectral irradiance of Shapiro et al. (2011), from which typical fluxes in bandpasses similar to those used for stars can be extracted, allows a direct comparison between solar and stellar cycles both in time and in amplitude. Time-frequency diagrams for the Sun in near- $B$ and $-V$ Johnson passbands, are plotted in Fig. 3.

In the left panel of Fig. 3 the temporal evolution of the solar cycles are shown as if the measurements would have been made in $B$ band. The amplitude of the Gleissberg cycle is naturally stronger during the Maunder (when the Schwabe cycle nearly disappeared) and Dalton (when the Schwabe cycle had very low amplitude) minima, whereas during the last 170 years the Schwabe cycle dominates. The right panel of Fig. 3 shows that in the $V$-band there is a domination of the Gleissberg cycle in the entire time-frequency diagram covering 400 years with a very weak signal of the Schwabe cycle in the latter two centuries. Note that the amplitudes are very small, the total change (min-max) is about 0.018 magnitude in $B$ and less, than 0.005 magnitude in $V$. In case of ground-based
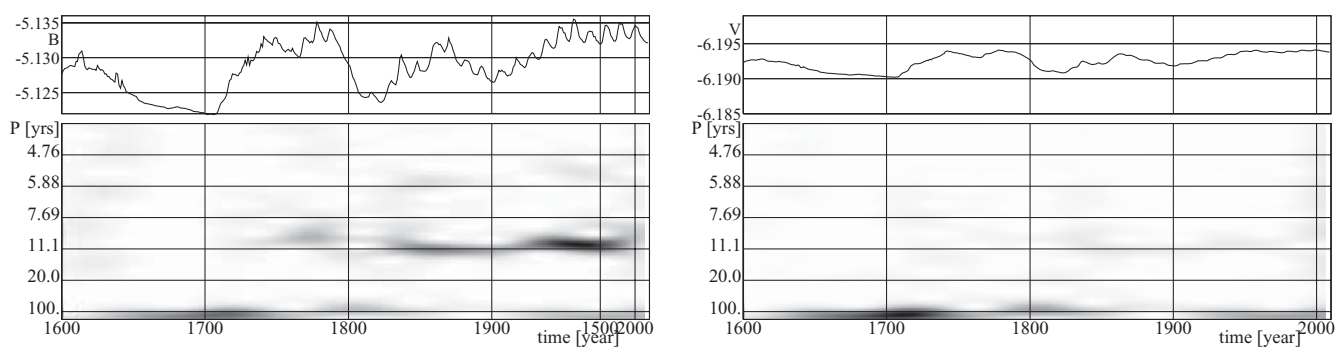

Figure 3. Time-frequency diagrams of the Sun, close to Johnson B (left) and V (right) bandpasses, similar to those traditionally used in stellar photometry. The relative amplitudes of the different cycles are well seen. 
observations of stars such changes are equal to the measurement errors. For the more active stars in Fig. 2 one can see that the stellar cycle amplitudes can be of several tenths of a magnitude in the $V$ band. From a stellar point of view, if the Sun was measured through the $V$ filter for decades, its brightness would seem to be constant, suitable to be used as a comparison star.

The existence of approximate $B V R$ data for the Sun allows another comparison to other active stars. As the area of the spots and faculae in view are changing due to stellar rotation and cycle phases, the stars also change their colors and, as inferred from this, their average surface temperatures. Spot-dominated stars are redder when fainter, while faculae-dominated stars are bluer when more activity (including spots) are present. Examples for both are found e.g., in Messina (2008), who identified e.g., V775 Her as spot-dominated and UX Ari as faculae-dominated. This latter type is a rare example of an active star being bluer when fainter.

Two color index vs. brightness diagrams of the Sun on Fig. 4 are based on the spectral reconstruction of Shapiro et al. (2011). The magnitudes have zero-point offsets. Therefore, we plotted the differences from the mean values of the actual data since we are looking for systematic changes only. The figure shows that the Sun belongs to the spot-dominated type of stars, although its variability is very small. Preminger et al. (2011), from full-disk images of the Sun through filters similar to the Strömgren $b, y$ filters also concluded that the Sun is spot-dominated in the visible continuum. Note, that in Fig. 4 as well, as in all similar diagrams of stars, data are from different phases of rotations and (multiple) cycles. However, all these flux modulations have the same origin, i.e., they are manifestations of the magnetic activity of stars, on different timescales and with different amplitudes.

The dominance of spots or faculae originate from the variable contribution of these features. The color index variation reflects temperature changes as well; when e.g., the facular area decreases faster than the spotted one the star becomes fainter (decreased relative contribution from the brighter component) and redder (less hotter area), as measured in the visible. In the high energy bandpasses the Sun looks like other stars with comparable parameters - see Judge and Thompson (2012) and references therein. However, measuring the Sun in the conventional bandpasses used for the stars is not easy and this was not systematically done, except for those data presented by Preminger et al. (2011). The latter authors call attention to the problem of accurate determination of the quiet-Sun flux: at high level of the solar activity low contrast bright features modify the flux of the quiet regions but this remains unnoticed. We would like to emphasize that
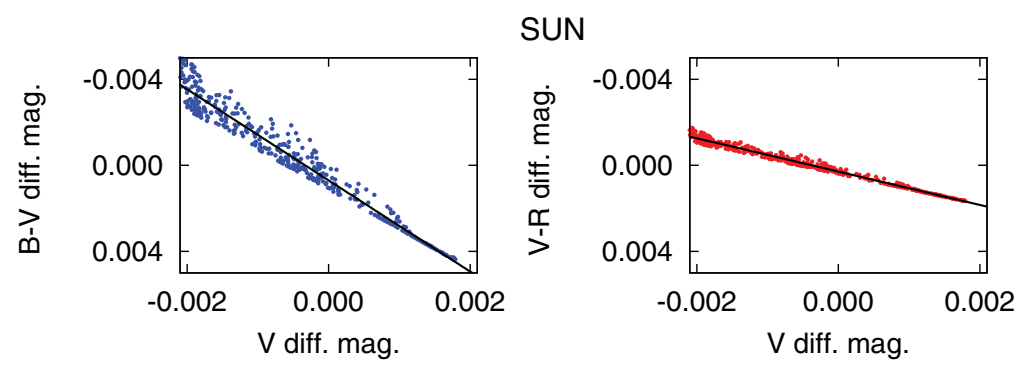

Figure 4. Solar brightness vs. temperature: relations between $B-V$ and $V-R$ color indices vs. $V$ relative magnitudes of the Sun based on Shapiro and co-workers' (Shapiro et al. 2011) spectral reconstruction. The fluxes are transformed to magnitudes, and the deviation from the mean values are plotted. As the majority of active stars, the Sun seems to be redder when fainter, i.e. spot-dominated, although the amplitudes are small. 

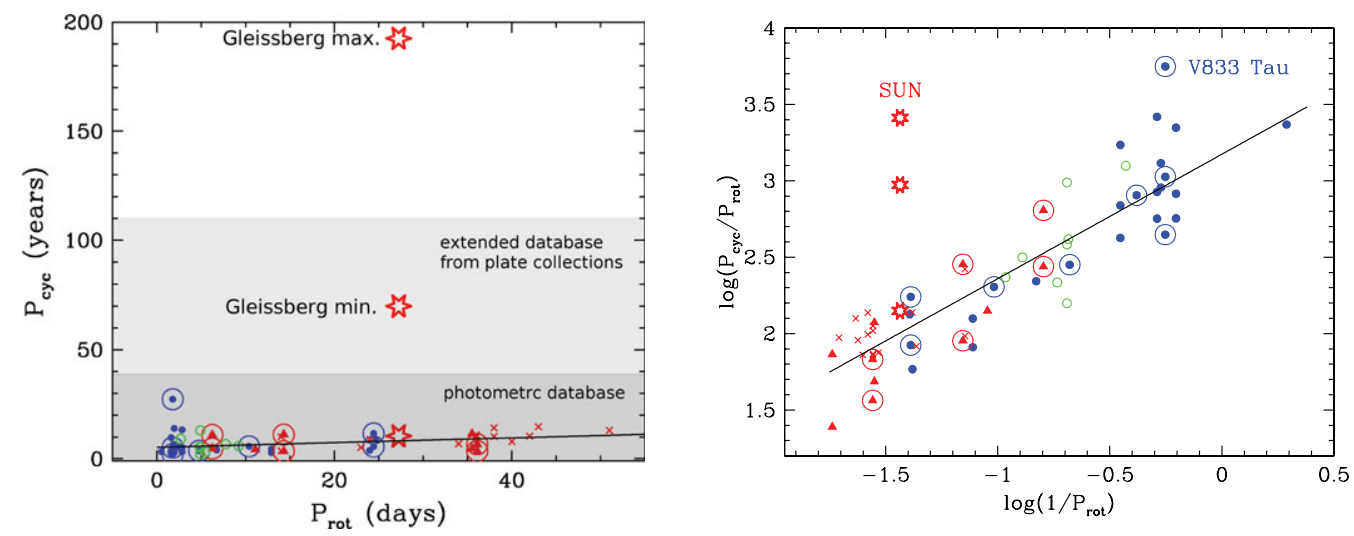

Figure 5. Stellar and solar cycles in the function of the rotational (or orbital) periods. Left: the dark grey area shows the length of a typical stellar dataset from photometry, and the light grey area at 110 years is the maximum length of stellar data extended using photographic data. Right: $\log -\log$ representation of the cycle lengths normalised with the rotational periods as function of the inverse of the rotational periods. Solid symbols represent results from Oláh et al. (2009), circled ones among those are representative of different rotational rates. Open circles and crosses are from the literature (Frick et al. 2004; Messina \& Guinan 2002).

when one compares the behaviors of the Sun and the stars, the same kinds of measurement should be used.

\section{Discussion and Conclusions}

Although it is interesting to study the multiple cycles and their changes of active stars and the Sun on their own, it is also important to see if there is any relation to physical parameters. The most evident measure at hand is the rotation of the objects. As discussed before, the lengths of the datasets are of crucial importance for identifying cycles. On the left panel of Fig. 5 we plot a rotation period vs. cycle length diagram for a sample of stars with the best cycle determinations currently exist. The two shaded areas show the lengths of the average photometric dataset (dark grey), and the maximum data record extended by archival photographic measurements (light grey). The moral of this figure is that the Gleissberg cycle of the Sun could at most be guessed from a dataset of the same length than the longest one available for stars. Additionally, the Sun, measured from a greater distance, with filters that are routinely used for stars, should have a light variation of at least 10-30 times higher than in fact has, for a detectable cycle!

An increase of the cycle lengths is seen towards longer rotational periods (Fig. 5, left panel). Baliunas et al. (1996) already found a loose relation between rotation and cycle length expressed in a way we plot the right panel of Fig. 5, i.e., logarithm of the cycle length normalized to the rotation period plotted as a function of the logarithm of the inverse rotation period. The slope of such a relation is related to the dynamo number (see Baliunas et al. (1996) for details). The relation is weak but it is the same for solar type, slowly rotating stars, dwarfs and giants, single stars and binary components. To quantify such a relation needs time - studying activity cycles of stars is only be possible with the help of routinely working automated telescopes, which have been successfully operating since decades and should continue to do so. 


\section{Acknowledgements}

Thanks are due to Z. Kolláth and A. Shapiro for useful discussions, and for the referee, M. Güdel for useful remarks. Support from the organizers of the meeting is much appreaciated. This work was supported by the Hungarian Research grant OTKA K-081421.

\section{References}

Baliunas, S., Nesme-Ribes, E., Sokoloff, D., \& Soon, W. 1996 ApJ 460, 848

Brown, B. P., Miesch, M. S., Browning, M. K. et al. 2011 ApJ 731, 69B

Frick, P., Soon, W., Popova, E., \& Baliunas, S. 2004 New Astron. 9, 599

Judge, P. G. \& Thompson, M. J. 2012 this proceedings

Kolláth, Z. \& Oláh, K. 2009, A\&\&A 501, 695

Kolláth, Z., Oláh, K., \& van Driel-Gesztelyi, L. 2011, this proceedings

Messina, S. \& Guinan, E. F. 2008, A\&A 393, 255

Messina, S. 2008, A\&A 480, 495

Oláh, K., Kolláth, Z., Granzer, T. et al. 2009, A\&̈A 501, 703

Preminger, D., Chapman, G. A., \& Cookson, A. M. 2011, ApJ 739, L45

Shapiro, A. I., Schmutz, W., Rozanov, E. et al. 2011, A\&A 529A, 67S

Strassmeier, K. G., Boyd, L. J., Epand, D. H., \& Granzer, Th. 1997, PASP 109, 697

Wilson, O. C. 1968, ApJ 153, 221 